\title{
Scrutinizing and Improving Impossible Differential Attacks: Applications to CLEFIA, Camellia, LBlock and SIMON*
}

\author{
Christina Boura ${ }^{1}$, María Naya-Plasencia ${ }^{2}$ and Valentin Suder ${ }^{2}$ \\ 1 Versailles Saint-Quentin-en-Yvelines University, France \\ christina.boura@prism.uvsq.fr \\ ${ }^{2}$ Inria, France \\ \{Maria.Naya_Plasencia,Valentin.Suder\}@inria.fr
}

\begin{abstract}
Impossible differential cryptanalysis has shown to be a very powerful form of cryptanalysis against block ciphers. These attacks, even if extensively used, remain not fully understood because of their high technicality. Indeed, numerous are the applications where mistakes have been discovered or where the attacks lack optimality. This paper aims in a first step at formalizing and improving this type of attacks and in a second step at applying our work to block ciphers based on the Feistel construction. In this context, we derive generic complexity analysis formulas for mounting such attacks and develop new ideas for optimizing impossible differential cryptanalysis. These ideas include for example the testing of parts of the internal state for reducing the number of involved key bits. We also develop in a more general way the concept of using multiple differential paths, an idea introduced before in a more restrained context. These advances lead to the improvement of previous attacks against well known ciphers such as CLEFIA-128 and Camellia, while also to new attacks against 23-round LBlock and all members of the Simon family.
\end{abstract}

Keywords: block ciphers, impossible differential attacks, CLEFIA, Camellia, LBlock, Simon.

\section{Introduction}

Impossible differential attacks were independently introduced by Knudsen 21] and Biham et al. [5]. Unlike differential attacks [6] that exploit differential paths of high probability, the aim of impossible differential cryptanalysis is to use differentials that have a probability of zero to occur in order to eliminate the key candidates leading to such impossible differentials.

The first step in an impossible differential attack is to find an impossible differential covering the maximum number of rounds. This is a procedure that

\footnotetext{
* Partially supported by the French Agence Nationale de la Recherche through the BLOC project under Contract ANR-11-INS-011.
} 
has been extensively studied and there exist algorithms for finding such impossible differentials efficiently 20199]. Once such a maximum-length impossible differential has been found and placed, one extends it by some rounds to both directions. After this, if a candidate key partially encrypts/decrypts a given pair to the impossible differential, then this key certainly cannot be the right one and is thus rejected. This technique provides a sieving of the key space and the remaining candidates can be tested by exhaustive search.

Despite the fact that impossible differential cryptanalysis has been extensively employed, the key sieving step of the attack does not seem yet fully understood. Indeed, this part of the procedure is highly technical and many parameters have to be taken into consideration. Questions that naturally arise concern the way to choose the plaintext/ciphertext pairs, the way to calculate the necessary data to mount the attack, the time complexity of the overall procedure as well as which are the parameters that optimize the attack. However, no simple and generalized way for answering these questions has been provided until now and the generality of most of the published attacks is lost within the tedious details of each application. The problems that arise from this approach is that mistakes become very common and attacks become difficult to verify. Errors in the analysis are often discovered and as we demonstrate in the next paragraph, many papers in the literature present flaws. These flaws include errors in the computation of the time or the data complexity, in the analysis of the memory requirements or of the complexity of some intermediate steps of the attacks. We can cite many such cases for different algorithms, as shown in Table 1. Note however, that the list of flaws presented in this table is not exhaustive.

Table 1. Summary of flaws in previous impossible differential attacks on CLEFIA-128, Camellia, LBlock and Simon. Symbol $\boldsymbol{x}$ means that the attack does not work, while $\checkmark$ says that the corrected attacks work. Error type (1) is when the data complexity is higher than the codebook, error type (2) shows a big computation flaw, error type (3) stands for small complexity flaws, while error type (4) is if the attack cannot be verified without implementation.

\begin{tabular}{|c|c|c|c|c|c|}
\hline Algorithm & \# rounds & Ref. & \begin{tabular}{|c|} 
Type \\
of error
\end{tabular} & $\begin{array}{c}\text { Repaira- } \\
\text { bility }\end{array}$ & $\begin{array}{c}\text { Where } \\
\text { discovered }\end{array}$ \\
\hline $\begin{array}{l}\text { CLEFIA-128 without } \\
\text { without whit. layers }\end{array}$ & $\overline{14}$ & 36 & $\overline{(1)}$ & $\overline{\bar{x}}$ & $\overline{113}$ \\
\hline CLEFIA-128 & 13 & 30 & $(4)$ & - & 7] \\
\hline $\begin{array}{l}\text { Camellia without } \\
F L / F L^{-1} \text { layers }\end{array}$ & 12 & 34 & $(2)$ & $x$ & $\begin{array}{l}\text { this paper, similar } \\
\text { problem as 33. }\end{array}$ \\
\hline Camellia-128 & 12 & 33 & $(2)$ & $x$ & 25 \\
\hline \begin{tabular}{|c|} 
Camellia-128/192/256 \\
without $F L / F L^{-1}$ layers \\
\end{tabular} & $11 / 13 / 14$ & 23 & $(3)$ & $\sqrt{ }$ & 34 \\
\hline LBlock & 22 & 26 & $(3)$ & $\sqrt{ }$ & 27 \\
\hline SIMON (all versions) & \begin{tabular}{|c|}
$14 / 15 / 15 / 16 / 16 /$ \\
$19 / 19 / 22 / 22 / 22$ \\
\end{tabular} & 3 & $(1)$ & $x$ & Table 1 of 3 ] \\
\hline SIMON (all versions) & $13 / 15 / 17 / 20 / 25$ & \begin{tabular}{|l|l|l|}
1 & 2 \\
\end{tabular} & $(2)$ & $x$ & this paper \\
\hline
\end{tabular}


Instances of such flaws can for example be found in analyses of the cipher CLEFIA. CLEFIA is a lightweight 128-bit block cipher developed by SONY in 2007 [28] and adopted as an international ISO/IEC 29192 standard in lightweight cryptography. This cipher has attracted the attention of many researchers and numerous attacks have been published so far on reduced round versions [31 32 230 24 298 8 . Most of these attacks rely on impossible differential cryptanalysis. However, as pointed out by the designers of CLEFIA [14, some of these attacks seem to have flaws, especially in the key filtering phase. We can cite here a recent paper by Blondeau [7] that challenges the validity of the results in [30], or a claimed attack on 14 rounds of CLEFIA-128 [36], for which the designers of CLEFIA showed that the necessary data exceeds the whole codebook [13. Another extensively analyzed cipher is the ISO/IEC 18033 standard Camellia, designed by Mitsubishi and NTT [4. Among the numerous attacks presented against this cipher, some of the more successful ones rely on impossible differential cryptanalysis 3433222523 . In the same way as for CLEFIA, some of these attacks were detected to have flaws. For instance, the attack from 33] was shown in 25] to be invalid. We discovered a similar error in the computation that invalidated the attack of [34]. Also, 34] reveals small flaws in [23]. Errors in impossible differential attacks were also detected for other ciphers. For example, in a cryptanalysis against the lightweight block cipher LBlock [26], the time complexity revealed to be incorrectly computed [27. Another problem can be found in 3], where the data complexity is higher than the amount of data available in the block cipher SIMON, or in 112, where some parameters are not correctly computed. During our analysis, we equally discovered problems in some attacks that do not seem to have been pointed out before. In addition to all this, the more the procedure becomes complicated, the more the approach lacks optimality. To illustrate this lack of optimality presented in many attacks we can mention a cryptanalysis against 22-round LBlock [18], that could easily be extended to 23 rounds if a more optimal approach had been used to evaluate the data and time complexities, as well as an analysis of Camellia [22] which we improve in Section 4 .

The above examples clearly show that impossible differential attacks suffer from the lack of a unified and optimized approach. For this reason, the first aim of our paper is to provide a general framework for dealing with impossible differential attacks. In this direction, we provide new generic formulas for computing the data, time and memory complexities. These formulas take into account the different parameters that intervene into the attacks and provide a highly optimized way for mounting them. Furthermore, we present some new techniques that can be applied in order to reduce the data needed or to reduce the number of key bits that need to be guessed. In particular we present a new method that helps reducing the number of key bits to be guessed by testing instead some bits of the internal state during the sieving phase. This technique has some similarities with the methods introduced in [1517, however important differences exist as both techniques are applied in a completely different context. In addition to this, we apply and develop the idea of multiple impossible differentials, intro- 
duced in 32, to obtain more data for mounting our attacks. To illustrate the strength of our new approach we consider Feistel constructions and we apply the above ideas to a number of block ciphers, namely CLEFIA, Camellia, LBlock and Simon.

More precisely, we present an attack as well as different time/data trade-offs on 13-round CLEFIA-128 that improve the time and data complexity of the previous best known attack 25] and improvements in the complexity of the best known attacks against all versions of Camellia [22]. In addition, in order to demonstrate the generality of our method, we provide the results of our attacks against 23-round LBlock and all versions of the SIMON block cipher. The attack on LBlock is the best attack so far in the single-key setting 1 , while our attacks on Simon are the best known impossible differential attacks for this family of ciphers and the best attacks in general for the three smaller versions of SimON.

Summary of Our Attacks. We present here a summary of our results on the block ciphers CLEFIA-128, Camellia, LBlock and SIMON and compare them to the best impossible differential attacks known for the four analyzed algorithms. This summary is given in Table 2 , where we point out with a ' $*$ ' if the mentioned attack is the best cryptanalysis result on the target cipher or not, i.e. by the best known attack we consider any attack reaching the highest number of rounds, and with the best complexities among them.

The rest of the paper is organized as follows. In Section 2 we present a generic methodology for mounting impossible differential attacks, provide our complexity formulas and show new techniques and improvements for attacking a Feistellike block cipher using impossible differential cryptanalysis. Section 3 is dedicated to the details of our attacks on CLEFIA and Section 4 presents our applications to all versions of Camellia. Due to lack of space, our applications on LBlock and the SIMON family of ciphers are given in the full version of this paper [1].

\section{Complexity Analysis}

We provide in this section a complexity analysis of impossible differential attacks against block ciphers as well as some new ideas that help improving the time and data complexities. We derive in this direction new generic formulas for the complexity evaluation of such attacks. The role of these formulas is twofold; on the one hand we aim at clarifying the attack procedure by rendering it as general as possible and on the other hand help at optimizing the time and data requirements. Establishing generic formulas should help mounting as well as verifying such attacks by avoiding the use of complicated procedures often leading to mistakes.

An impossible differential attack consists mainly of two general steps. The first one deals with the discovery of a maximum-length impossible differential, that is an input difference $\Delta_{X}$ and an output difference $\Delta_{Y}$ such that the probability

${ }^{1}$ In [12], an independent and simultaneous result on 23-round LBlock with worse time complexity was proposed. 
Table 2. Summary of the best impossible differential attacks on CLEFIA-128, Camellia, LBlock and SImON and presentation of our results. The presence of a '*' mentions if the current attack is the best known attack against the target cipher. Note here that we provide only the best of our results with respect to the time complexity. Other trade-offs can be found in the following sections. ${ }^{\dagger}$ see Section 4 for details.

\begin{tabular}{|c|c|c|c|c|c|}
\hline Algorithm & Rounds & Time & $\begin{array}{l}\text { Data } \\
(\mathrm{CP}) \\
\end{array}$ & $\begin{array}{l}\text { Memory } \\
\text { (Blocks) }\end{array}$ & Reference \\
\hline "CLEFIA-128 & $\overline{13}$ & $2^{121.2}$ & $2^{117.8}$ & $2^{86.8}$ & 24 \\
\hline using state-test technique & 13 & $2^{116.90}$ & $2^{116.33}$ & $2^{83.33}$ & Section 3 \\
\hline using multiple impossible differentials & 13 & $2^{122.26}$ & $2^{111.02}$ & $2^{82.60}$ & Section $3^{*}$ \\
\hline combining with state-test technique & 13 & $2^{116.16}$ & $2^{114.58}$ & $2^{83.16}$ & $11]^{*}$ \\
\hline Camellia-128 & 11 & $2^{122}$ & $2^{122}$ & $2^{98}$ & 22 \\
\hline & 11 & $2^{118.43}$ & $2^{118.4}$ & $2^{92.4}$ & Section $4^{*}$ \\
\hline Camellia-192 & 12 & $2^{187.2}$ & $2^{123}$ & $2^{155.41}$ & 22 \\
\hline & 12 & $2^{161.06}$ & $2^{119.7}$ & $2^{150.7}$ & Section $4^{*}$ \\
\hline Camellia-256 & 13 & $2^{251.1}$ & $2^{123}$ & $2^{203}$ & 22 \\
\hline & 13 & $2^{225.06}$ & $2^{119.71}$ & $2^{198.71}$ & Section $4^{*}$ \\
\hline Camellia-256 ${ }^{\dagger}$ & 14 & $2^{250.5}$ & $2^{120}$ & $2^{120}$ & 22 \\
\hline & 14 & $2^{220}$ & $2^{118}$ & $2^{173}$ & Section 4 \\
\hline$\overline{\overline{\text { LBlock }}}$ & $\overline{\overline{222}}$ & $2^{79.28}$ & $2^{58}$ & $2^{72.67}$ & 18 \\
\hline & 22 & $2^{71.53}$ & $2^{60}$ & $2^{59}$ & 1110 \\
\hline & 23 & $2^{74.06}$ & $2^{59.6}$ & $2^{74.6}$ & 11110] \\
\hline "SIMON32/64 & 19 & $\overline{2} 2^{62.56}$ & $\overline{2^{32}}$ & $2^{44}$ & $\overline{111]^{*}}$ \\
\hline SIMON48/72 & 20 & $2^{70.69}$ & $2^{48}$ & $2^{58}$ & $11]^{*}$ \\
\hline SIMON48/96 & 21 & $2^{94.73}$ & $2^{48}$ & $2^{70}$ & $11]^{*}$ \\
\hline SIMON64/96 & 21 & $2^{94.56}$ & $2^{64}$ & $2^{60}$ & 11 \\
\hline SIMON64/128 & 22 & $2^{126.56}$ & $2^{64}$ & $2^{75}$ & 11 \\
\hline SIMON96/96 & 24 & $2^{94.62}$ & $2^{94}$ & $2^{61}$ & 11 \\
\hline SimOn96/144 & 25 & $2^{190.56}$ & $2^{128}$ & $2^{77}$ & 11 \\
\hline SiMON128/128 & 27 & $2^{126.6}$ & $2^{94}$ & $2^{61}$ & 11 \\
\hline SIMON128/192 & 28 & $2^{190.56}$ & $2^{128}$ & $2^{77}$ & 11 \\
\hline SIMON128/256 & 30 & $2^{254.68}$ & $2^{128}$ & $2^{111}$ & 11 \\
\hline
\end{tabular}

that $\Delta_{X}$ propagates after a certain number of rounds, $r_{\Delta}$, to $\Delta_{Y}$ is zero. The second step, called the key sieving phase, consists in the addition of some rounds to potentially both directions. These extra added rounds serve to verify which key candidates partially encrypt (resp. decrypt) data to the impossible differential. As this differential is of probability zero, keys showing such behavior are clearly not the right encryption key and are thus removed from the candidate keys space.

We start by introducing the notation used in the rest of the paper. As in this work we are principally interested in the key sieving phase, we start our attack after a maximum impossible differential has been found for the target cipher. 


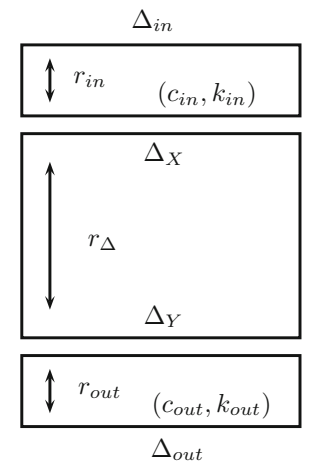

- $\Delta_{X}, \Delta_{Y}$ : input (resp. output) differences of the impossible differential.

$-r_{\Delta}$ : number of rounds of the impossible differential.

$-\Delta_{\text {in }}, \Delta_{\text {out }}$ : set of all possible input (resp. output) differences of the cipher.

$-r_{i n}$ : number of rounds of the differential $\operatorname{path}\left(\Delta_{X}, \Delta_{i n}\right)$.

- $r_{\text {out }}$ : number of rounds of the differential $\operatorname{path}\left(\Delta_{Y}, \Delta_{\text {out }}\right)$.

The differential $\left(\Delta_{X} \rightarrow \Delta_{\text {in }}\right)$ (resp. $\left.\left(\Delta_{Y} \rightarrow \Delta_{\text {out }}\right)\right)$ occurs with probability 1 while the differential $\left(\Delta_{X} \leftarrow \Delta_{\text {in }}\right)$ (resp. $\left.\left(\Delta_{Y} \leftarrow \Delta_{\text {out }}\right)\right)$ is verified with probability $\frac{1}{2^{c_{i n}}}$ (resp. $\frac{1}{2^{c_{\text {out }}}}$ ), where $c_{\text {in }}$ (resp. $c_{\text {out }}$ ) is the number of bit-conditions that have to be verified to obtain $\Delta_{X}$ from $\Delta_{\text {in }}$ (resp. $\Delta_{Y}$ from $\Delta_{\text {out }}$ ).

It is important to correctly determine the number of key bits intervening during an attack. We call this quantity information key bits. In an impossible differential attack, one starts by determining all the subkey bits that are involved in the attack. We denote by $k_{i n}$ the subset of subkey bits involved in the attack during the first $r_{\text {in }}$ rounds, and $k_{\text {out }}$ during the last $r_{\text {out }}$ ones. However, some of these subkey bits can be related between them. For example, two different subkey bits can actually be the same bit of the master key. Alternatively, a bit in the set can be some combination, or can be easily determined by some other bits of the set. The way that the different key bits in the target set are related is determined by the key schedule. The actual parameter that we need to determine for computing the complexity of the attacks is the information key bits intervening in total, that is from an information theoretical point of view, the $\log$ of the entropy of the involved key bits, that we denote by $\left|k_{\text {in }} \cup k_{\text {out }}\right|$.

We continue now by describing our attack scenario on $\left(r_{\text {in }}+r_{\Delta}+r_{\text {out }}\right)$ rounds of a given cipher.

\subsection{Attack Scenario}

Suppose that we are dealing with a block cipher of block size $n$ parametrized by a key $K$ of size $|K|$. Let the impossible differential be placed between the rounds $\left(r_{i n}+1\right)$ and $\left(r_{i n}+r_{\Delta}\right)$. As already said, the impossible differential implies that it is not feasible that an input difference $\Delta_{X}$ at round $\left(r_{i n}+1\right)$ propagates to an output difference $\Delta_{Y}$ at the end of round $\left(r_{i n}+r_{\Delta}\right)$. Thus, the goal is, for each given pair of inputs (and their corresponding outputs), to discard the keys that generate a difference $\Delta_{X}$ at the beginning of round $\left(r_{i n}+1\right)$ and at the same time, a difference $\Delta_{Y}$ at the output of round $\left(r_{i n}+r_{\Delta}\right)$. We need then enough pairs so that the number of non-discarded keys is significantly lower than the a priori total number of key candidates. 
Suppose that the first $r_{i n}$ rounds have an input truncated difference in $\Delta_{i n}$ and an output difference $\Delta_{X}$, which is the input of the impossible differential. Suppose that there are $c_{i n}$ bit-conditions that need to be verified so that $\Delta_{i n}$ propagates to $\Delta_{X}$ and $\left|k_{i n}\right|$ information key bits involved.

In a similar way, suppose that the last $r_{\text {out }}$ rounds have a truncated output difference in $\Delta_{\text {out }}$ and an input difference $\Delta_{Y}$, which is the output of the impossible differential. Suppose that there are $c_{\text {out }}$ bit-conditions that need to be verified so that $\Delta_{\text {out }}$ propagates to $\Delta_{Y}$ in the backward direction and $\left|k_{\text {out }}\right|$ information key bits involved.

We show next how to determine the amount of data needed for an attack.

\subsection{Data Complexity}

The probability that for a given key, a pair of inputs already satisfying the differences $\Delta_{\text {in }}$ and $\Delta_{\text {out }}$ verifies all the $\left(c_{\text {in }}+c_{\text {out }}\right)$ bit-conditions is $2^{-\left(c_{\text {in }}+c_{\text {out }}\right)}$. In other words, this is the probability that for a pair of inputs having a difference in $\Delta_{i n}$ and an output difference in $\Delta_{\text {out }}$, a key from the possible key set is discarded. Therefore, by repeating the procedure with $N$ different input (or output) pairs, the probability that a trial key is kept in the candidate keys set is $P=\left(1-2^{-\left(c_{\text {in }}+c_{\text {out }}\right)}\right)^{N}$.

There is not a unique strategy for choosing the amount of input (or output) pairs $N$. This choice principally depends on the overall time complexity, which is influenced by $N$, and the induced data complexity. Different trade-offs are therefore possible. A popular strategy, generally used by default is to choose $N$ such that only the right key is left after sieving. This amounts to choose $P$ as

$$
P=\left(1-2^{-\left(c_{\text {in }}+c_{\text {out }}\right)}\right)^{N}<\frac{1}{2^{\left|k_{\text {in }} \cup k_{\text {out }}\right|}} .
$$

In this paper we adopt a different approach that can help reducing the number of pairs needed for the attack and offers better trade-offs between the data and time complexity. More precisely, we permit smaller values of $N$. By proceeding like this, we will be probably left with more than one key in our candidate keys set and we will need to proceed to an exhaustive search among the remaining candidates, but the total time complexity of the attack will probably be much lower. In practice, we will start considering values of $N$ such that $P$ is slightly smaller than $\frac{1}{2}$ so to reduce the exhaustive search by at least one bit. The smallest value of $N$, denoted by $N_{\min }$, verifying

$$
P=\left(1-2^{-\left(c_{\text {in }}+c_{\text {out }}\right)}\right)^{N_{\min }} \simeq e^{-N_{\min } \times 2^{-\left(c_{\text {in }}+c_{\text {out }}\right)}}<\frac{1}{2}
$$

is approximately $N_{\min }=2^{c_{\text {in }}+c_{\text {out }}}$. Then we have to choose $N \geq N_{\min }$.

We provide then a solution for determining the cost of obtaining $N$ pairs such that their input difference belongs to $\Delta_{i n}$ and their output difference belongs 
to $\Delta_{\text {out }}$. To the best of our knowledge, this is the first generic solution to this problem. We evaluated this cost as

$$
C_{N}=\max \left\{\min _{\Delta \in\left\{\Delta_{\text {in }}, \Delta_{\text {out }}\right\}}\left\{\sqrt{N 2^{n+1-|\Delta|}}\right\}, N 2^{n+1-\left|\Delta_{\text {in }}\right|-\left|\Delta_{\text {out }}\right|}\right\} .
$$

A detailed explanation on how this formula is derived can be found in the full version of the paper [1]. The $\operatorname{cost} C_{N}$ represents also the amount of needed data. Obviously, as the size of the state is $n$, the following inequality, should hold:

$$
C_{N} \leq 2^{n}
$$

This inequality simply states that the total amount of data used for the attack cannot exceed the codebook. These conditions are not verified in several cases from [3], as well as in the corrected version of [36] which invalidates the corresponding attacks.

\subsection{Time and Memory Complexity}

We are going to detail now the computation of the time complexity of the attack. Note that the formulas that we are presenting in this section are the first generic formulas given for estimating the complexity of impossible differential attacks.

By following the early abort technique [23], the attack consists in storing the $N$ pairs and testing out step by step the key candidates, by reducing at each time the size of the remaining possible pairs. The time complexity is then determined by three quantities. The first term is the cost $C_{N}$, that is the amount of needed data (see Formula (1) for obtaining the $N$ pairs, where $N$ is such that $P<1 / 2$. The second term corresponds to the number of candidate keys $2^{\left|k_{\text {in }} \cup k_{\text {out }}\right|}$, multiplied by the average cost of testing the remaining pairs. For all the applications that we have studied, this cost can be very closely approximated by $\left(N+2^{\left|k_{\text {in }} \cup k_{\text {out }}\right|} \frac{N}{2^{c_{\text {in }}+c_{\text {out }}}}\right) C_{E}^{\prime}$, where $C_{E}^{\prime}$ is the ratio of the cost of partial encryption to the full encryption. Finally, the third term is the cost of the exhaustive search for the key candidates still in the candidate keys set after the sieving. By taking into account the cost of one encryption $C_{E}$, we conclude that the time complexity of the attack is

$$
T_{\text {comp }}=\left(C_{N}+\left(N+2^{\left|k_{\text {in }} \cup k_{\text {out }}\right|} \frac{N}{2^{c_{\text {in }}+c_{\text {out }}}}\right) C_{E}^{\prime}+2^{|K|} P\right) C_{E},
$$

where $C_{N}=\max \left\{\min _{\Delta \in\left\{\Delta_{\text {in }}, \Delta_{\text {out }}\right\}}\left\{\sqrt{N 2^{n+1-|\Delta|}}\right\}, N 2^{n+1-\left|\Delta_{\text {in }}\right|-\left|\Delta_{\text {out }}\right|}\right\}$, with $N$ such that $P=\left(1-1 /\left(2^{c_{\text {in }}+c_{\text {out }}}\right)\right)^{N}<1 / 2$ and where the last term corresponds to $2^{|K|-\left|k_{\text {in }} \cup k_{\text {out }}\right|} P 2^{\left|k_{\text {in }} \cup k_{\text {out }}\right|}$. Obviously, as we want the attack complexity to be smaller than the exhaustive search complexity, the above quantity should be smaller than $2^{|K|} C_{E}$.

It must be noted here that this is a minimum estimation of the complexity, that, in practice, and thanks to the idea of Section 2.4 it approximates really 
well the actual time complexity, as it can be seen in the applications, and in particular, in the tight correspondence shown between the LBlock estimation that we detail in 11 and the exact calculation from 10. The precise evaluation of $C_{E}^{\prime}$ (that is always smaller than 1) can only be done once the attack parameters are known. However, $C_{E}^{\prime}$ can be estimated quite by calculating the ratio between the active SBoxes during a partial encryption and the total number of SBoxes (thought it is not always the best approximation, it is a common practice).

Memory complexity. By using the early abort technique [23], the only elements that need to be stored are the $N$ pairs. Therefore, the memory complexity of the attack 2 is determined by $N$.

\subsection{Choosing $\Delta_{i n}, \Delta_{o u t}, c_{i n}$ and $c_{o u t}$}

We explain now, the two possible ways for choosing $\Delta_{\text {in }}, \Delta_{\text {out }}, c_{\text {in }}$ and $c_{\text {out }}$. For this, we introduce the following example that can be visualized in Figure 1 and where we consider an Sbox-based cipher. In this example, we will only talk about $\Delta_{\text {in }}$ and $c_{i n}$, however the approach for $\Delta_{\text {out }}$ and $c_{\text {out }}$ is identical.

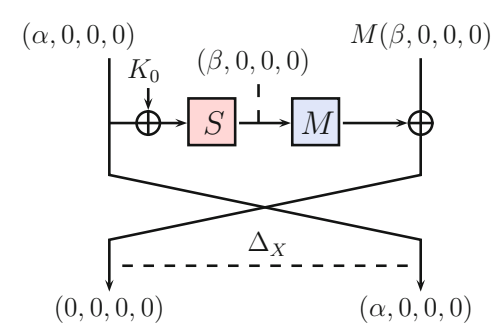

Fig. 1. Choosing $\Delta_{i n}$ and $c_{i n}$

Suppose that the state is composed of two branches of four nibbles each. The round function is composed of a non-linear layer $S$, seen as a concatenation of four Sboxes $S_{0}, S_{1}, S_{2}$ and $S_{3}$, followed by a linear layer $M$. There exist two different ways for choosing $\left|\Delta_{i n}\right|$ and $c_{i n}$ :

1. The most intuitive way is to consider $\left|\Delta_{i n}\right|=4+4$ and $c_{i n}=4$, as the size of $\alpha$ and of $\beta$ is 4 bits, and in the first round we want 4 bits to collide. In this case, for a certain key, the average probability that a pair taken out of the $2^{4+4} 2^{4+4-1}$ pairs belonging to $\Delta_{i n}$ leads to $\Delta_{X}$ is $2^{-4}$.

2. In general, the difference $\alpha$ can take $2^{4}-1$ different values. However, each value can be associated by the differential distribution table of the Sbox $S_{0}$

$\overline{{ }^{2} \text { If } N>2^{\mid k_{\text {in }}} \cup k_{\text {out }} \mid}$ we could store the discarded key candidates instead, this is rarely the case. Thus, we can consider a memory complexity of $\min \left\{N, 2^{\left|k_{\text {in }} \cup k_{\text {out }}\right|}\right\}$. 
to $2^{3}$ output differences on average 3 , so the possibilities for the difference $\beta$ are limited to $2^{3}$. Therefore, we can consider that $\left|\Delta_{i n}\right| \approx 4+3$. But, in this case $c_{i n}=3$, as for each input pair belonging to the $2^{4+3} 2^{4+3-1}$ possible ones, there exist on average 2 values that make the differential transition $\alpha \rightarrow \beta$ possible (instead of 1 in the previous case).

We can see, by using the generic formulas of Section 2.3, that both cases induce practically the same time complexity, as the difference in $N$ compensates with the difference in $c_{i n}+c_{\text {out }}$. However, the memory complexity, given by $N$, is slightly better in case 2. Furthermore, case 2, in which a preliminary pairs filtering is done, allows to reduce the average cost of using the early abort technique 23 .

In several papers, for example in 33 and 23 , the second case is followed. However, its application is partial (either for the input or the output part) and this with no apparent reason. Note however, that in these papers, the associated $c_{\text {out }}$ was not always correctly computed and sometimes, 8-bit conditions were considered when 7-bit conditions should have been accounted for. For reasons of simplicity, we will consider case 1 in our applications and check afterwards the actual memory needed.

\subsection{Using Multiple Impossible Differentials to Reduce the Data Complexity}

We explain in this section a method to reduce the data complexity of an attack. This method is inspired by the notion of multiple impossible differentials that was introduced by Tsunoo et al. 32 and applied to 12-round CLEFIA-128. The idea in this technique is to consider at once several impossible differentials, instead of just one. We assume, as done in [16, that the differences in $\Delta_{\text {in }}$ (and in $\Delta$ out) lie in a closed set. There are two ways in which this can be a priori done:

1. Take rotated versions of a certain impossible differential. We call $n_{\text {in }}$ the number of different input pattern differences generated by the rotated versions of the chosen impossible differential.

2. When the middle conditions have several impossible combinations, we can consider the same first half of the differential path together with a rotated version of the second one, in a way to get a different impossible differential. We call $n_{\text {out }}$ the number of different output pattern differences generated by the rotated versions of the second part of the path that we will consider. For the sake of simplicity and without loss of generality we will only consider the case of rotating the second half of the path.

It is important to point out that for our analysis to be valid, in both cases the number of conditions associated to the impossible differential attack should stay the same. Both cases can be translated into a higher amount of available

3 This quantity depends on the Sbox. In this example, we consider that all four Sboxes have good cryptographic properties. 
data by redefining two quantities, $\left|\Delta_{\text {in }}^{\prime}\right|$ and $\left|\Delta_{\text {out }}^{\prime}\right|$, that will take the previous roles of $\left|\Delta_{\text {in }}\right|$ and $\left|\Delta_{\text {out }}\right|$,

$$
\left|\Delta_{\text {in }}^{\prime}\right|=\left|\Delta_{\text {in }}\right|+\log _{2}\left(n_{\text {in }}\right) \text { and }\left|\Delta_{\text {out }}^{\prime}\right|=\left|\Delta_{\text {out }}\right|+\log _{2}\left(n_{\text {out }}\right) .
$$

$\left|\Delta_{\text {in }}^{\prime}\right|$ is the log of the total size of the set of possible input differences, and $\left|\Delta_{\text {out }}^{\prime}\right|$ is the $\log$ of the total size of the set of possible output differences.

In this case, the data complexity $C_{N}$ is computed with the corrected values for the input sizes and is, as can be easily seen, smaller than if only one path had been used. The time complexity remains the same, except for the $C_{N}$ term. Indeed, the middle term of Formula (2) remains the same, as for a given pair, the number of key bits involved stays $2^{\left|k_{\text {in }} \cup k_{\text {out }}\right|}$. Equally, as the number of involved possible partial keys is $n_{\text {in }} n_{\text {out }} 2^{\left|k_{\text {in }} \cup k_{\text {out }}\right|}$, the last term of Formula (2) is now

$$
\frac{2^{|K|}}{n_{\text {in }} \cdot n_{\text {out }} 2^{\left|k_{\text {in }} \cup k_{\text {out }}\right|}}\left(P \cdot n_{\text {in }} \cdot n_{\text {out }} \cdot 2^{\left|k_{\text {in }} \cup k_{\text {out }}\right|}\right)=2^{|K|} P
$$

and so also stays the same.

In Section 3 we present our attacks on CLEFIA. In part of these attacks, we use multiple impossible differentials to reduce the data complexity. Besides, this technique shows particularly useful for mounting attacks on some versions of the Simon family for which there is not enough available data to mount a valid attack with the traditional method.

\subsection{Introducing the State-Test Technique}

We introduce now a new method that consists in making a test for some part of the internal state instead of guessing the necessary key bits for computing it. This somewhat reminds the techniques presented in [15]17] in the context of meet-in-the-middle attacks. However, the technique that we present in this section, and that we call the state-test technique is different since it consists in checking the values of the internal state to verify if we can discard all the involved candidates.

Very often during the key filtering phase of impossible differential attacks, the size of the internal state that needs to be known is smaller than the number of key bits on which it depends. As we will see, focusing on the values that a part of the state can take permits to eliminate some key candidates without considering all the values for the involved key bits. The state-test technique works by fixing $s$ bits of the plaintexts, which allows us to reduce the number of information key bits by $s$. We will explain how this method works by a small example.

Consider a 32-bit Feistel construction, where each branch can be seen as a concatenation of four nibbles (see Figure 2). Suppose that the round function is composed of a non-linear layer $S$, seen as a concatenation of four 4-bit invertible Sboxes $\left(S_{0}, S_{1}, S_{2}, S_{3}\right)$ and of a linear layer $M$ on $\mathbb{F}_{2^{4}}$. We suppose for this example that the branch number of $M$, that is the minimal number of active Sboxes in any two consecutive rounds, is less than 5 . Let $\Delta_{X}=(\alpha, 0,0,0) \mid(0,0,0,0)$ be the input difference of the impossible differential, placed at the end of the second 
round and let $\Delta_{i n}=(*, *, *, 0) \mid(*, *, *, *)$ be the difference at the input of the block cipher. Note however that in reality, the leftmost side of $\Delta_{\text {in }}$ only depends on a 4 -bit non-zero difference $\delta$, i.e. $\Delta_{i n}=M(\delta, 0,0,0) \mid(*, *, *, *)$.

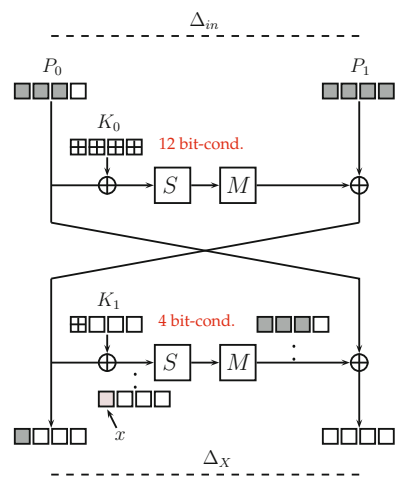

Fig. 2. Grey color stands for nibbles with non-zero difference. Hatched key nibbles correspond to the part of the subkeys that have to be guessed. The nibble $x$ is the part of the state on which we apply the state-test technique.

As can be seen in Figure 2, there are in total 4 active Sboxes and thus there are $c_{i n}=16$ conditions that have to be verified in order to have a transition from $\Delta_{i n}$ to $\Delta_{X}$. Therefore, the first step is to collect $N$ pairs such that $P=$ $\left(1-2^{-\left(c_{\text {in }}+c_{\text {out }}\right)}\right)^{N}=\left(1-2^{-c_{\text {in }}}\right)^{N}=\left(1-2^{-16}\right)^{N}<\frac{1}{2}$. The exact value of $N$ will be chosen in a way to obtain the best trade-off for the complexities. Before describing the new method, we start by explaining how this attack would have worked in the classical way. As we can see in Figure 2, there are $3 \times 4$ bits that have to be guessed $\left(K_{0,0}, K_{0,1}\right.$ and $\left.K_{0,2}\right)$ in order to verify the conditions on the first round and there are $2 \times 4$ bits that have to be guessed $\left(K_{0,3}\right.$ and $\left.K_{1,0}\right)$ in order to verify the conditions on the second round.

Therefore, for all $N$ pairs, one starts by testing all the $2^{4}$ possible values for the first nibble of $K_{0}$. After this first guess, $N \times 2^{-4}$ pairs remain in average, as there are 4-bit conditions that need to be verified by the guess through the first round. Then one continues by testing the second and the third nibble of $K_{0}$ and finally the last nibble of $K_{0}$ and the first nibble of $K_{1}$. At each step, the amount of data remaining is divided by $2^{4}$. To summarize, we have $\left|k_{\text {in }} \cup k_{\text {out }}\right|=\left|k_{\text {in }}\right|=20$ and $2^{c_{\text {in }}+c_{\text {out }}}=2^{c_{\text {in }}}=2^{4} 2^{4} 2^{4} 2^{4}$. Then Formula (2) can be used to evaluate the time complexity of the attack as

$$
\left(C_{N}+\left(N+2^{20} \frac{N}{2^{16}}\right) C_{E}^{\prime}+2^{20} P 2^{|K|-20}\right) C_{E} .
$$

We will see now how the state-test technique applies to this example and how it permits to decrease the time complexity. Consider the first nibble of the left 
part of the state after the addition of the subkey $K_{1}$. We denote this nibble by $x$. Note that mathematically, $x$ can be expressed as

$$
\begin{aligned}
x & =K_{1,0} \oplus P_{1,0} \oplus M\left(S\left(K_{0} \oplus P_{0}\right)\right)_{0} \\
x \oplus P_{1,0} & =K_{1,0} \oplus m_{0} S_{0}\left(K_{0,0} \oplus P_{0,0}\right) \oplus m_{1} S_{1}\left(K_{0,1} \oplus P_{0,1}\right) \\
& \oplus m_{2} S_{2}\left(K_{0,2} \oplus P_{0,2}\right) \oplus m_{3} S_{3}\left(K_{0,3} \oplus P_{0,3}\right),
\end{aligned}
$$

where the $m_{i}$ 's are coefficients in $\mathbb{F}_{2}^{4}$.

Suppose now that for all pairs, we fix the last $s=4$ bits of $P_{0}$ to the same constant value. One can verify that this is a reasonable assumption, as by fixing this part of the inputs we still have enough data to mount the attack. Then one starts as before, by guessing the first three nibbles of $K_{0}$. After this 12-bit guess, approximately $N \times 2^{-12}$ pairs remain. We know for each pair the input and output differences of the Sbox of the second round as the needed part of $K_{0}$ has been guessed. Therefore, by a simple lookup at the differential distribution table of the involved Sbox, we obtain one value for $x$ that verifies the second round conditions in average per pair (about half of the time the transition is not possible, whereas for the other half we find two values). Equation (44) becomes

$$
\begin{aligned}
& x \oplus P_{1,0} \oplus m_{0} S_{0}\left(K_{0,0} \oplus P_{0,0}\right) \oplus m_{1} S_{1}\left(K_{0,1} \oplus P_{0,1}\right) \oplus m_{2} S_{2}\left(K_{0,2} \oplus P_{0,2}\right) \\
& =K_{1,0} \oplus m_{3} S_{3}\left(K_{0,3} \oplus P_{0,3}\right),
\end{aligned}
$$

where the left side of Equation (5), that we denote by $x^{\prime}$, is known for each pair.

Thus, for each guess of $\left(K_{0,0}, K_{0,1}, K_{0,2}\right)$, we construct a table of size $N \times 2^{-12}$, where we store these values of $x^{\prime}$. The last and more important step consists now in looking if all $2^{4}$ possible values of $x^{\prime}$ appear in the table. Note here, that as $N \geq 2^{16}$, the size of the table is necessarily greater than or equal to $2^{4}$.

Since $P_{0,3}$ is fixed, the only unknown values in Equation (5) are $K_{1,0}$ and $K_{0,3}$. If all values for $x^{\prime}$ are in the table and since $S_{3}$ is a permutation, for any choice of $K_{1,0}$ and any choice of $K_{0,3}$, there will always exist (at least) one pair such that $K_{1,0} \oplus m_{3} S_{3}\left(K_{0,3} \oplus P_{0,3}\right)$ is in the table, leading thus to the impossible differential.

As a conclusion, we know that if $x^{\prime}$ takes all the possible values in the table, we can remove the keys composed by the guessed value $\left(K_{0,0}, K_{0,1}, K_{0,2}\right)$ from the candidate keys set, as for all the values of $\left(K_{1,0}, K_{0,3}\right)$, they would imply the impossible differential. If instead, $x^{\prime}$ does not take all the possible values for a certain value of $\left(K_{0,0}, K_{0,1}, K_{0,2}\right)$, we can test this partial key combined to all the possibilities of the remaining key bits that verify Equation (5) for the missing $x^{\prime}$, as they belong to the remaining key candidates.

The main gain of the state-test technique is that it decreases the number of information key bits and therefore the time complexity. For instance, 
in this example, the variable $x^{\prime}$ can be seen as 4 information key bits 4 instead of $2 \times 4$ key bits we had to guess in the classic approach (the bits of $K_{0,3}$ and of $\left.K_{1,0}\right)$. We have $s=4$ less bits to guess thanks to the $s=4$ bits of the plaintext that we have fixed. Thus the time complexity in this case becomes

$$
\left(C_{N}+\left(N+2^{20-4} \frac{N}{2^{16}}\right) C_{E}^{\prime}+2^{20-4} P 2^{|K|-(20-4)}\right) C_{E} .
$$

One can see now by comparing Equations (6) and (3) that the time complexity is lower with the state-test technique, than with the trivial method. Indeed, the first and the third term of the Equations (6) and (3) remain the same, while the second term is lower in Equation (6). Finally, note that the probability $P$ for a key to be still in the candidate keys set remains the same as before. Indeed, during the attack we detect all and the same candidate keys for which none of the $N$ pairs implies the impossible differential, which are the same candidate keys that we would have detected in a classic attack.

We would like to note here that we have implemented the state-test technique on a toy cipher, having a structure similar to the one that we introduced in this section, and we have verified its correctness.

Application of the state-test technique in parallel for decreasing the probability $P$. An issue that could appear with this technique is that as we have to fix a part of the plaintexts, $s$ bits, the amount of data available for computing the $N$ pairs is reduced. The probability $P$ associated to an attack is the probability for a key to remain in the candidate keys set. When the amount of available data is small, the number of pairs $N$ that we can construct is equally small and thus the probability $P$ is high. In such a situation, the dominant term of the time complexity (Formula (2) ), is in general the third one, i.e. $2^{|K|} P$.

More precisely, we need the sum of $\log _{2}\left(C_{N}\right)$ and $s$, the number of plaintext bits that we fix, to be less than or equal to the block size. This limits the size of $N$ that we can consider, leading to higher probabilities $P$, and could lead, sometimes, to higher time complexities. To avoid this, one can repeat the attack in parallel for several different values, say $Y$, of the fixed part of the plaintext. In this case, the data and memory needed are multiplied by $Y$. On the other hand, repeating the attack in parallel permits to detect more efficiently if a guessed key could be the right one. Indeed, for a guessed key, only if none of the tables constructed as described above contains all the values for $x^{\prime}$, one can test if this guessed key is the correct one.

To summarize, by repeating the state-test technique in parallel, we multiply the available data by $Y$, as well as the available pairs, and since the attack is done $Y$ times in parallel, the probability $P$ becomes $P^{Y}$. The probability decreases

${ }^{4}$ Note that we could, equivalently, consider all possible values of $x^{\prime}$ in the last step, and consider the associated remaining pairs table, that would have a size of $N 2^{-16}$ (empty if the key is a good candidate, not empty otherwise), obtaining the same key candidates of 16 bits, 12 from $\left(K_{0,0}, K_{0,1}, K_{0,2}\right)$ and 4 information key bits from $x^{\prime}$, with the same complexity as in the previously described method. 
much faster than the data or the other terms of the time complexity increase. Therefore, the Formula (2) becomes in this case:

$$
\left(C_{N} \times Y+\left(N \times Y+2^{\left|k_{i n} \cup k_{\text {out }}\right|-s} \frac{N \times Y}{2^{c_{\text {in }}+c_{\text {out }}}}\right) C_{E}^{\prime}+2^{|K|} P^{Y}\right) C_{E} .
$$

In Section 3, we are going to see an application of this technique to 13-round CLEFIA-128, and at the end of Section 4 we show an application on Camellia-256.

\section{Application to CLEFIA}

CLEFIA is a lightweight 128-bit block cipher designed by Shirai et al. in 2007 [28] and based on a 4-branch generalized Feistel network. It supports keys of size 128, 192 or 256 bits and the total number of iterations, say $R$, depends on the key size. More precisely, $R=18$ for the 128-bit version, while $R=22$ and $R=26$ for the two following variants. A key-scheduling algorithm is used to generate $2 R$ round keys $R K_{0}, \ldots, R K_{2 R-1}$ and 4 whitening keys $W K_{0}, \ldots, W K_{3}$. The whitening keys are XORed to the right branches of the first and the last round. CLEFIA's round function design can be visualized in Figure 3. For a more complete description of the specifications one can refer to [28.

We describe now several attacks against 13-round CLEFIA-128.

\subsection{Impossible Differential Cryptanalysis of 13-round CLEFIA-128}

The authors of 31 noticed that a difference on the internal state of CLEFIA of the form $P^{i}=0_{32}\left|0_{32}\right| 0_{32} \mid A$ cannot lead to a difference $P^{i+9}=0_{32}\left|0_{32}\right| B \mid 0_{32}$ after 9 rounds, where $A$ and $B$ are 4-byte vectors for which only one byte in a different position is active (e.g. $A=\left(\alpha, 0_{8}, 0_{8}, 0_{8}\right)$ and $\left.B=\left(0_{8}, \beta, 0_{8}, 0_{8}\right)\right)$. We use this same 9 -round impossible differential and place it between rounds 3 and 11. Therefore, for our attack, $r_{\text {in }}=r_{\text {out }}=2$ and $r_{\Delta}=9$, as in [24].
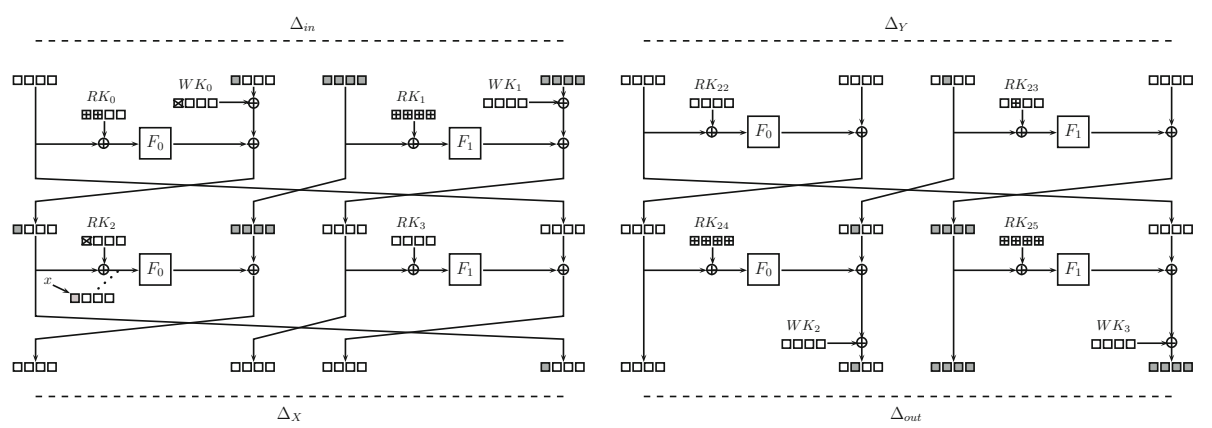

Fig. 3. The attack on CLEFIA-128. Grey color stands for bytes with a non-zero difference, while hatched bytes are the subkey bytes that have to be guessed. 
The differential placed on the top and at the bottom of the impossible differential are depicted in Figure 3. We describe now the parameters for our cryptanalysis of 13-round CLEFIA-128. As can be seen in Figure 3 there are $c_{\text {in }}+c_{\text {out }}=40+40$ bit-conditions that need to be verified so that the difference in the plaintexts $\Delta_{i n}=0_{32}\left|\left(*_{8}, 0_{8}, 0_{8}, 0_{8}\right)\right| M_{0}\left(*_{8}, 0_{8}, 0_{8}, 0_{8}\right) \mid *_{32}$ propagates to $\Delta_{X}=0_{32}\left|0_{32}\right| 0_{32} \mid\left(\alpha, 0_{8}, 0_{8}, 0_{8}\right)$ and the difference in the ciphertexts $\Delta_{\text {out }}=$ $0_{32}\left|\left(0_{8}, *_{8}, 0_{8}, 0_{8}\right)\right| M_{1}\left(0_{8}, *_{8}, 0_{8}, 0_{8}\right) \mid *_{32}$ propagates to $\Delta_{Y}=0_{32}\left|0_{32}\right|\left(0_{8}, \beta, 0_{8}, 0_{8}\right)$ $\mid 0_{32}$. In this way, $\left|\Delta_{\text {in }}\right|=\left|\Delta_{\text {out }}\right|=48$.

Following the complexity analysis of Section 2, we need to construct at least $N_{\text {min }}=2^{80}$ pairs. The cost to construct these pairs is

$$
C_{N_{\min }}=\max \left\{\sqrt{2^{80} 2^{129-48}}, 2^{80} 2^{129-48-48}\right\}=2^{113} .
$$

Using the state-test technique. We use now the state-test technique, described in Section 2.6 to test the 8 bits of the internal state denoted by $x$ in Figure 3 , instead of guessing the whole subkey $R K_{0}$ and the XOR of the leftmost byte of $R K_{2}$ and $W K_{0}$. For doing this, we need to fix part of the 32 leftmost bits of the plaintexts. As the number of needed data is $C_{N_{\min }}=2^{113}$, we can fix at most $128-113=15$ bits. However, as each Sbox is applied to 8 bits, we will only fix one byte of this part of the plaintexts. We will guess then 24 bits of the subkey $R K_{0}$ which are situated on the other bytes.

During a classical attack procedure, we would need to guess 32 bits of $R K_{1}, 32$ bits of $R K_{0}$ and 8 bits of $R K_{2} \oplus W K_{0}$, thus $k_{i n}=72$. We would also need to guess 8 bits of $R K_{23} \oplus W K_{2}, 32$ bits of $R K_{24}$ and 32 bits of $R K_{25}$, therefore $k_{\text {out }}=72$. However, the subkeys $R K_{1}$ and $R K_{24}$ share 22 bits in common. As a consequence, the number of information key bits would be $\left|k_{\text {in }} \cup k_{\text {out }}\right|=72+72-22=122$. As we will fix 8 bits of the plaintexts, according to Section 2.6, it is the same to say that there will be $\left|k_{\text {in }} \cup k_{\text {out }}\right|-8=122-8=114$ bits to test. The time complexity of our attack, computed using Formula (2) is then

$$
\left(C_{N}+\left(N+2^{114} \frac{N}{2^{80}}\right) \frac{18}{104}+2^{128} P\right) C_{E},
$$

where the fraction $18 / 104$ is the ratio of the cost of partial encryption to the full encryption. Since our attack needs at least $2^{113}$ plaintexts and since we fixed 8 bits out of them, we have $128-113-8=7$ bits of freedom for building structures.

Among all possible trade-offs with respect to the amount of data, the best time complexity is $2^{116.90} C_{E}$ with $2^{83.33}$ pairs built from $2^{116.33}$ plaintexts.

Using multiple impossible differentials. The authors of [31 noticed that there exist several different 9-round impossible differentials, see [31, Table 1]. In [32, multiple impossible differentials were used to attack 12 rounds of CLEFIA-128. Here, we will apply our formalized approach of this idea presented in Section 2.5, to reduce the data complexity of the attack on 13 rounds of CLEFIA-128.

We use the $n_{\text {in }}=2 \times 4$ different inputs to the impossible differentials, that is $P^{i}=0_{32}|A| 0_{32} \mid 0_{32}$ and $P^{i}=0_{32}\left|0_{32}\right| 0_{32} \mid A$, where $A$ can take a difference on only 
one of the four possible bytes. For each one of them, there are $n_{\text {out }}=3$ different output impossible differences $P^{i+9}=0_{32}\left|0_{32}\right| B \mid 0_{32}$ after 9 rounds, where $B$ has only one byte active in a different position than the active byte in $A$. We have now $\left|\Delta_{\text {in }}^{\prime}\right|=\left|\Delta_{\text {in }}\right|+\log _{2}(8)=48+3$ and $\left|\Delta_{\text {out }}^{\prime}\right|=\left|\Delta_{\text {out }}\right|+\log _{2}(3)=48+1.58$. Since the bit-conditions remain unchanged, $c_{\text {in }}+c_{\text {out }}=80$, the minimal number of pairs needed for the attack to work is $N_{\min }=2^{80}$. For this number of pairs, we need $C_{N_{\min }}=2^{113-4.58}=2^{108.42}$ plaintexts. The number of information key bits is $\left|k_{\text {in }} \cup k_{\text {out }}\right|=122$. We have then $\left(C_{N}+\left(N+2^{122} \frac{N}{2^{80}}\right) \frac{18}{104}+P 2^{128}\right) C_{E}$. Among all the possible trade-offs with respect to the amount of data, the best time complexity we obtained is $2^{122.26} C_{E}$ with $2^{82.6}$ pairs built from $2^{111.02}$ plaintexts. Recall here that the aim of this approach was to reduce data complexity. Thus, in this attack the gain on the data complexity is the important part5.

In the full version of this paper [1] we show how to combine the state-testtechnique together with multiple differentials in order to reduce at the same time the time and the data complexity for the attacks on CLEFIA-128.

\section{Applications to Camellia}

Camellia is a 128-bit block cipher designed by Aoki et. al. in 2000 [4. It is a Feistel-like construction where two key-dependent layers $F L$ and $F L^{-1}$ are applied every 6 rounds to each branch. Whitening keys are equally applied to the first and the last round of the cipher. There exist three different versions of the cipher, that we note Camellia-128, Camellia-192 and Camellia-256, depending on the key size used. The number of iterations is 18 for the 128-bit version and 24 for the other two versions. A detailed description of Camellia's structure can be found in the full version of the paper. For further details, one can refer to [4].

Previous Cryptanalysis. Camellia is since 2005 an international ISO/IEC standard and has therefore attracted a lot of attention from the cryptographic community. Since Camellia has a particular design, involving the so-called $F L / F L^{-1}$ layers, its cryptanalysis can be classified in several categories. Some attacks consider the $F L / F L^{-1}$ functions, while others do not take them into consideration. Equally, some attacks take into account the whitening keys, whereas others don't and finally all attacks do not start from the same round. The best attacks on Camellia in terms of the number of rounds and the complexities are those presented in [22, Section 4.2].

Here we start by presenting improvements of the best attacks that include the $F L / F L^{-1}$ layers and the whitening keys. Next we build an attack using the state-test technique on 14-round Camellia-256 starting from the first round but without the $F L / F L^{-1}$ layers and the whitening keys.

Improvements. We improve here the complexities of the previous attacks that take into account the $F L / F L^{-1}$ layers and the whitening keys on all three

\footnotetext{
${ }^{5}$ In [24], the authors used a loose approximation for $C_{E}^{\prime}$, as $C_{E}^{\prime}=1 / 104$.
} 
versions of Camellia. By using the complexity analysis introduced in Section 2 , we can optimize the complexities of the corresponding attacks from 22. Note that we use for this the same parameters as in 22 . The parameters of our attacks on 11-round Camellia-128, 12-round Camellia-192 and 13-round Camellia-256 are depicted in Table 3 . As can be seen in Table 2 the time complexity of our improved attack on Camellia- 128 is $2^{118.43} C_{E}$, with data complexity $2^{118.4}$ and memory complexity $2^{92.4}$. For Camellia-192, the time, data and memory complexities are $2^{161.06} C_{E}, 2^{119.7}$ and $2^{150.7}$ respectively, while for Camellia-256 the corresponding complexities are $2^{225.06} C_{E}, 2^{119.71}$ and $2^{198.71}$.

Table 3. Attack parameters against all versions of Camellia

\begin{tabular}{|c||cc||ccc||cc||c||}
\hline Algorithm & $\left|\Delta_{\text {in }}\right|$ & $\left|\Delta_{\text {out }}\right|$ & $r_{\text {in }}$ & $r_{\text {out }}$ & $r_{\Delta}$ & $c_{\text {in }}$ & $c_{\text {out }}$ & $\left|k_{\text {in }} \cup k_{\text {out }}\right|$ \\
\hline Camellia-128 & 23 & 80 & 1 & 2 & 8 & 32 & 57 & 96 \\
\hline Camellia-192 & 80 & 80 & 2 & 2 & 8 & 73 & 73 & 160 \\
\hline Camellia-256 & 80 & 128 & 2 & 3 & 8 & 73 & 121 & 224 \\
\hline
\end{tabular}

Using the State-Test Technique on Camellia-256. We provide here an impossible differential attack on Camellia-256 without $F L / F L^{-1}$ layers and whitening keys by using the state-test technique. Note here, that unlike all previous attacks not starting from the first round in order to take advantage of the key schedule asymmetry, our attack starts from the first round of the cipher. It covers 14 rounds of Camellia-256 which is, to the best of our knowledge, the highest number of rounds attacked for this version. In [22] another attack on 14-round Camellia-256 with $F L / F L^{-1}$ and whitening keys is presented, however, as said before, it does not start from the first round and it uses a specific property of the key schedule at the rounds where it is applied.

In this attack, we consider the same 8-round impossible differential as in 25] and we add $4+2$ rounds such that $r_{i n}=4, r_{\text {out }}=2$ and $r_{\Delta}=8$. We have $\left|\Delta_{i n}\right|=$ $128,\left|\Delta_{\text {out }}\right|=56, c_{\text {in }}=120$ and $c_{\text {out }}=48$. Then we need at least $N_{\min }=2^{168}$ plaintexts pairs. The amount of data needed to construct these pairs is $C_{N_{\min }}=$ $\max \left\{\sqrt{2^{168} 2^{129-128}}, 2^{168} 2^{129-184}\right\}=2^{113}$. There remain then $128-113=15$ bits of freedom. Thus, we can fix $s=8$ bits on the ciphertexts to apply the state-test technique on the 8 bits of the internal state at the penultimate round. The number of information key bits is $\left|k_{\text {in }} \cup k_{\text {out }}\right|=227-8=219$ since there are 45 bits shared between the subkeys with respect to the key schedule. The best attack is obtained with $N=2^{118}$ pairs. In this case, the time complexity is $2^{220} C_{E}$, the data complexity is $2^{118}$ plaintexts and the memory is $2^{118}$.

\section{Conclusion}

To start with, we have proposed in this paper a generic vision of impossible differential attacks with the aim of simplifying and helping the construction 
and verification of this type of cryptanalysis. Until now, these attacks were very tedious to mount and even more to verify, and so, very often flaws appeared in the computations. We believe that our objective has been successfully reached, as it can be seen by the high amount of new improved attacks that we have been able to propose, as well as by all the different possible trade-offs for each one of them, something that would be near to unthinkable prior to our work.

Next, the generic and clear vision of impossible differential attacks has allowed us to discover and propose new ideas for improving these attacks. In particular, we have proposed the state-test technique, that allows to reduce the number of key bits involved in the attack, and so to reduce the time complexity. We have also formalized and adapted to our generic scenario the notion introduced in 32 of multiple impossible differentials. This option allows reducing the data complexity. Finally, we have proposed several applications for different variants of the Feistel ciphers CLEFIA, Camellia, LBlock and Simon, providing in most of the cases, the best known attack on reduced-round versions of these ciphers.

We hope that these results will simplify and improve future impossible attacks on Feistel ciphers, as well as their possible combination with other attacks. For instance, in [35] a combination of impossible differential with linear attacks is proposed. We haven't verified these results, but this direction could be promising.

\section{References}

1. Abed, F., List, E., Lucks, S., Wenzel, J.: Differential and linear cryptanalysis of reduced-round SIMON. Cryptology ePrint Archive, Report 2013/526 (2013)

2. Abed, F., List, E., Wenzel, J., Lucks, S.: Differential Cryptanalysis of roundreduced Simon and Speck. In: FSE 2014. LNCS. Springer (to appear, 2014)

3. Alkhzaimi, H.A., Lauridsen, M.M.: Cryptanalysis of the SIMON Family of Block Ciphers. Cryptology ePrint Archive, Report 2013/543 (2013)

4. Aoki, K., Ichikawa, T., Kanda, M., Matsui, M., Moriai, S., Nakajima, J., Tokita, T.: Camellia: A 128-Bit Block Cipher Suitable for Multiple Platforms - Design and Analysis. In: Stinson, D.R., Tavares, S. (eds.) SAC 2000. LNCS, vol. 2012, pp. 39-56. Springer, Heidelberg (2001)

5. Biham, E., Biryukov, A., Shamir, A.: Cryptanalysis of Skipjack Reduced to 31 Rounds Using Impossible Differentials. In: Stern, J. (ed.) EUROCRYPT 1999. LNCS, vol. 1592, pp. 12-23. Springer, Heidelberg (1999)

6. Biham, E., Shamir, A.: Differential Cryptanalysis of DES-like Cryptosystems. In: Menezes, A., Vanstone, S.A. (eds.) CRYPTO 1990. LNCS, vol. 537, pp. 2-21. Springer, Heidelberg (1991)

7. Blondeau, C.: Improbable Differential from Impossible Differential: On the Validity of the Model. In: Paul, G., Vaudenay, S. (eds.) INDOCRYPT 2013. LNCS, vol. 8250, pp. 149-160. Springer, Heidelberg (2013)

8. Bogdanov, A., Geng, H., Wang, M., Wen, L., Collard, B.: Zero-Correlation Linear Cryptanalysis with FFT and Improved Attacks on ISO Standards Camellia and CLEFIA. In: Lange, T., Lauter, K., Lisoněk, P. (eds.) SAC 2013. LNCS, vol. 8282, pp. 306-323. Springer, Heidelberg (2013)

9. Bouillaguet, C., Dunkelman, O., Fouque, P.-A., Leurent, G.: New Insights on Impossible Differential Cryptanalysis. In: Miri, A., Vaudenay, S. (eds.) SAC 2011. LNCS, vol. 7118, pp. 243-259. Springer, Heidelberg (2012) 
10. Boura, C., Minier, M., Naya-Plasencia, M., Suder, V.: Improved Impossible Differential Attacks against Round-Reduced LBlock. Cryptology ePrint Archive, Report 2014/279 (2014)

11. Boura, C., Naya-Plasencia, M., Suder, V.: Scrutinizing and Improving Impossible Differential Attacks: Applications to CLEFIA, Camellia, LBlock and Simon (Full Version). Cryptology ePrint Archive, Report 2014/699 (2014)

12. Chen, J., Futa, Y., Miyaji, A., Su, C.: Impossible differential cryptanalysis of LBlock with concrete investigation of key scheduling algorithm. Cryptology ePrint Archive, Report 2014/272 (2014)

13. CLEFIA Design Team. Comments on the impossible differential analysis of reduced round CLEFIA presented at Inscrypt 2008 (January 8, 2009)

14. CLEFIA Design Team. Private communication (May 2014)

15. Dunkelman, O., Sekar, G., Preneel, B.: Improved Meet-in-the-Middle Attacks on Reduced-Round DES. In: Srinathan, K., Rangan, C.P., Yung, M. (eds.) INDOCRYPT 2007. LNCS, vol. 4859, pp. 86-100. Springer, Heidelberg (2007)

16. Gilbert, H., Peyrin, T.: Super-Sbox Cryptanalysis: Improved Attacks for AESLike Permutations. In: Hong, S., Iwata, T. (eds.) FSE 2010. LNCS, vol. 6147, pp. 365-383. Springer, Heidelberg (2010)

17. Isobe, T., Shibutani, K.: Generic Key Recovery Attack on Feistel Scheme. In: Sako, K., Sarkar, P. (eds.) ASIACRYPT 2013, Part I. LNCS, vol. 8269, pp. 464-485. Springer, Heidelberg (2013)

18. Karakoç, F., Demirci, H., Harmancı, A.E.: Impossible Differential Cryptanalysis of Reduced-Round LBlock. In: Askoxylakis, I., Pöhls, H.C., Posegga, J. (eds.) WISTP 2012. LNCS, vol. 7322, pp. 179-188. Springer, Heidelberg (2012)

19. Kim, J., Hong, S., Lim, J.: Impossible differential cryptanalysis using matrix method. Discrete Mathematics 310(5), 988-1002 (2010)

20. Kim, J., Hong, S., Sung, J., Lee, C., Lee, S.: Impossible Differential Cryptanalysis for Block Cipher Structures. In: Johansson, T., Maitra, S. (eds.) INDOCRYPT 2003. LNCS, vol. 2904, pp. 82-96. Springer, Heidelberg (2003)

21. Knudsen, L.R.: DEAL - A 128-bit cipher. Technical Report, Department of Informatics, University of Bergen, Norway (1998)

22. Liu, Y., Li, L., Gu, D., Wang, X., Liu, Z., Chen, J., Li, W.: New Observations on Impossible Differential Cryptanalysis of Reduced-Round Camellia. In: Canteaut, A. (ed.) FSE 2012. LNCS, vol. 7549, pp. 90-109. Springer, Heidelberg (2012)

23. Lu, J., Kim, J., Keller, N., Dunkelman, O.: Improving the Efficiency of Impossible Differential Cryptanalysis of Reduced Camellia and MISTY1. In: Malkin, T. (ed.) CT-RSA 2008. LNCS, vol. 4964, pp. 370-386. Springer, Heidelberg (2008)

24. Mala, H., Dakhilalian, M., Shakiba, M.: Impossible Differential Attacks on 13Round CLEFIA-128. J. Comput. Sci. Technol. 26(4), 744-750 (2011)

25. Mala, H., Shakiba, M., Dakhilalian, M., Bagherikaram, G.: New Results on Impossible Differential Cryptanalysis of Reduced-Round Camellia-128. In: Jacobson Jr., M.J., Rijmen, V., Safavi-Naini, R. (eds.) SAC 2009. LNCS, vol. 5867, pp. 281-294. Springer, Heidelberg (2009)

26. Minier, M., Naya-Plasencia, M.: A Related Key Impossible Differential Attack Against 22 Rounds of the Lightweight Block Cipher LBlock. Inf. Process. Lett. 112(16), 624-629 (2012)

27. Minier, M., Naya-Plasencia, M.: Private communication (May 2013)

28. Shirai, T., Shibutani, K., Akishita, T., Moriai, S., Iwata, T.: The 128-Bit Blockcipher CLEFIA (Extended Abstract). In: Biryukov, A. (ed.) FSE 2007. LNCS, vol. 4593, pp. 181-195. Springer, Heidelberg (2007) 
29. Tang, X., Sun, B., Li, R., Li, C.: Impossible differential cryptanalysis of 13-round CLEFIA-128. Journal of Systems and Software 84(7), 1191-1196 (2011)

30. Tezcan, C.: The Improbable Differential Attack: Cryptanalysis of Reduced Round CLEFIA. In: Gong, G., Gupta, K.C. (eds.) INDOCRYPT 2010. LNCS, vol. 6498, pp. 197-209. Springer, Heidelberg (2010)

31. Tsunoo, Y., Tsujihara, E., Shigeri, M., Saito, T., Suzaki, T., Kubo, H.: Impossible Differential Cryptanalysis of CLEFIA. In: Nyberg, K. (ed.) FSE 2008. LNCS, vol. 5086, pp. 398-411. Springer, Heidelberg (2008)

32. Tsunoo, Y., Tsujihara, E., Shigeri, M., Suzaki, T., Kawabata, T.: Cryptanalysis of CLEFIA using multiple impossible differentials. In: Information Theory and Its Applications, ISITA 2008, pp. 1-6 (2008)

33. Wu, W., Zhang, L., Zhang, W.: Improved Impossible Differential Cryptanalysis of Reduced-Round Camellia. In: Avanzi, R.M., Keliher, L., Sica, F. (eds.) SAC 2008. LNCS, vol. 5381, pp. 442-456. Springer, Heidelberg (2009)

34. Wu, W., Zhang, W., Feng, D.: Impossible Differential Cryptanalysis of ReducedRound ARIA and Camellia. J. Comput. Sci. Technol. 22(3), 449-456 (2007)

35. Yuan, Z., Li, X., Liu, H.: Impossible Differential-Linear Cryptanalysis of ReducedRound CLEFIA-128. Cryptology ePrint Archive, Report 2013/301 (2013)

36. Zhang, W., Han, J.: Impossible Differential Analysis of Reduced Round CLEFIA. In: Yung, M., Liu, P., Lin, D. (eds.) Inscrypt 2008. LNCS, vol. 5487, pp. 181-191. Springer, Heidelberg (2009) 